\title{
The Orbiting Carbon Observatory (OCO-2): spectrometer performance evaluation using pre-launch direct sun measurements
}

\author{
C. Frankenberg ${ }^{1}$, R. Pollock ${ }^{1}$, R. A. M. Lee ${ }^{1}$, R. Rosenberg ${ }^{1}$, J.-F. Blavier ${ }^{1}$, D. Crisp ${ }^{1}$, C. W. O'Dell ${ }^{2}$, G. B. Osterman ${ }^{1}$, \\ C. Roehl ${ }^{3}$, P. O. Wennberg ${ }^{3}$, and D. Wunch ${ }^{3}$ \\ ${ }^{1}$ Jet Propulsion Laboratory, California Institute of Technology, Pasadena, CA, USA \\ ${ }^{2}$ Colorado State University, Fort Collins, CO, USA \\ ${ }^{3}$ California Institute of Technology, Pasadena, CA, USA
}

Correspondence to: C. Frankenberg (christian.frankenberg@jpl.nasa.gov)

Received: 9 July 2014 - Published in Atmos. Meas. Tech. Discuss.: 28 July 2014

Revised: 15 November 2014 - Accepted: 22 November 2014 - Published: 14 January 2015

\begin{abstract}
The Orbiting Carbon Observatory-2 (OCO-2), launched on 2 July 2014, is a NASA mission designed to measure the column-averaged $\mathrm{CO}_{2}$ dry air mole fraction, $X_{\mathrm{CO}_{2}}$. Towards that goal, it will collect spectra of reflected sunlight in narrow spectral ranges centered at $0.76,1.6$ and $2.0 \mu \mathrm{m}$ with a resolving power $(\lambda / \Delta \lambda)$ of 20000 . These spectra will be used in an optimal estimation framework to retrieve $X_{\mathrm{CO}_{2}}$. About 100000 cloud free soundings of $X_{\mathrm{CO}_{2}}$ each day will allow estimates of net $\mathrm{CO}_{2}$ fluxes on regional to continental scales to be determined. Here, we evaluate the OCO-2 spectrometer performance using pre-launch data acquired during instrument thermal vacuum tests in April 2012. A heliostat and a diffuser plate were used to feed direct sunlight into the OCO-2 instrument and spectra were recorded. These spectra were compared to those collected concurrently from a nearby high-resolution Fourier Transform Spectrometer that was part of the Total Carbon Column Observing Network (TCCON). Using the launch-ready OCO-2 calibration and spectroscopic parameters, we performed total column scaling fits to all spectral bands and compared these to TCCON results. On 20 April, we detected a $\mathrm{CO}_{2}$ plume from the Los Angeles basin at the JPL site with strongly enhanced short-term variability on the order of $1 \%$ (3-4 ppm). We also found good $(<0.5 \mathrm{ppm})$ inter-footprint consistency in retrieved $X_{\mathrm{CO}_{2}}$. The variations in spectral fitting residuals are consistent with signal-to-noise estimates from instrument calibration, while average residuals are systematic and mostly attributable to remaining errors in our knowledge of the $\mathrm{CO}_{2}$ and $\mathrm{O}_{2}$ spectroscopic parameters. A few remaining inconsistencies observed during the tests may be attributable
\end{abstract}

to the specific instrument setup on the ground and will be re-evaluated with in-orbit data.

\section{Introduction}

After the launch failure of OCO (Crisp et al., 2004) in 2009, the National Aeronautics and Space Administration (NASA) authorized the development of OCO-2, which was successfully launched on 2 July 2014 at 2:56 a.m. Pacific daylight time (PDT) from Vandenberg Air Force Base on the California coast. To achieve its mission goal, OCO-2 spectra must enable retrievals of column averaged atmospheric $\mathrm{CO}_{2}$ (denoted as $X_{\mathrm{CO}_{2}}$ ) with an accuracy of $1 \mathrm{ppm}$ or better. A typical algorithm for the retrieval of $X_{\mathrm{CO}_{2}}$ (Bösch et al., 2006; Butz et al., 2009; O'Dell et al., 2012) concurrently employs three spectral bands, centered around $0.76 \mu \mathrm{m}\left(\mathrm{O}_{2} \mathrm{~A}\right.$-band $)$, $1.61 \mu \mathrm{m}$ (weak $\mathrm{CO}_{2}$ band) and $2.06 \mu \mathrm{m}$ (strong $\mathrm{CO}_{2}$ band). By using this multi-channel approach, $X_{\mathrm{CO}_{2}}$, surface albedos, as well as aerosol properties can be retrieved concurrently.

\section{OCO-2 instrument overview}

This paper describes spectral fitting results from direct sun observations collected with the OCO-2 instrument during thermal vacuum tests (TVACs) in April 2012 at the Jet Propulsion Laboratory (JPL) in Pasadena, California. We compare the instrument performance with measurements 


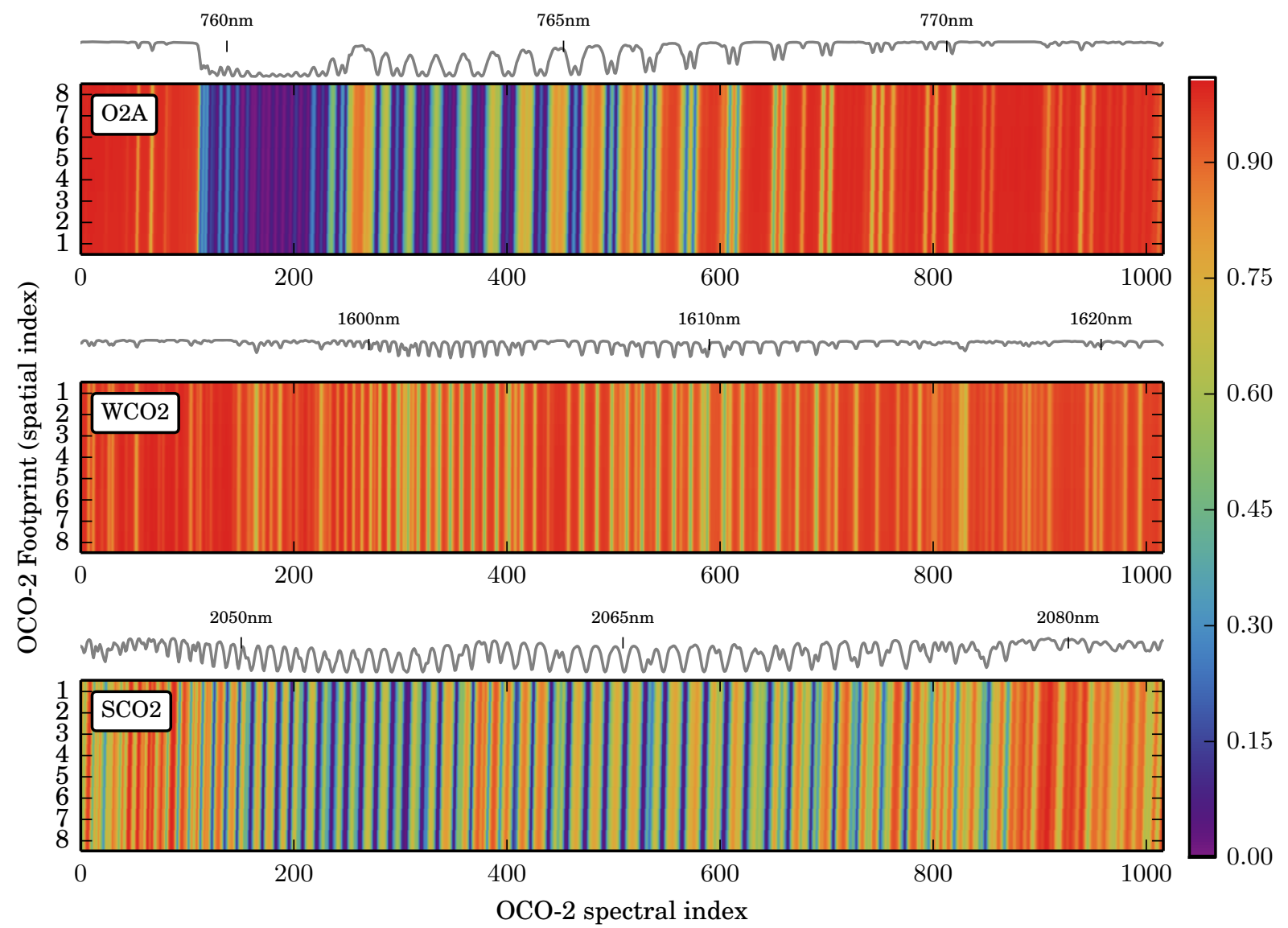

Figure 1. OCO-2 transmission spectra from TVAC direct-sun measurements at an air mass factor (AMF) of $\approx 2.5$. Transmission levels from 0 to 1 are color-coded for the $\mathrm{O} 2 \mathrm{~A}, \mathrm{WCO} 2$ and $\mathrm{SCO} 2$ bands, from top to bottom. The $x$ axis represents the spectral dimension (1016 elements) of the OCO-2 focal plane and the $y$ axis the spatial (160 elements co-added to eight individual footprints). As a guide for the eye, a spectral cross section of the center footprint is shown on top of each image, including wavelength indicators at the top. The detectors are slightly tilted with respect to the slit orientation for the $\mathrm{O}_{2}$ A-band in particular, but also for the strong $\mathrm{CO}_{2}$ band, causing a stronger footprint dependence of the dispersion coefficients.

collected by a high-resolution Fourier transform spectrometer (FTS) from the Total Carbon Column Observing Network (TCCON) (Washenfelder et al., 2006; Wunch et al., 2011) that was located at the same altitude, $\approx 200 \mathrm{~m}$ from the TVAC facilities at JPL. By using actual retrievals from the OCO-2 data, we can evaluate retrieval-relevant instrument properties that will help guide users of the OCO-2 spectra. While retrievals using direct sun observations are simpler than retrievals using reflected sunlight from space, these measurements from OCO-2 provide an end-to-end description of the combined impact of the OCO-2 instrument calibration as well as other aspects of the retrieval, such as the spectrally dependent gas absorption cross sections. Detailed descriptions of the instrument calibration can be found in Rosenberg et al. (2015); O'Dell et al. (2011) for radiometric calibration and Lee et al. (2015); Day et al. (2011) for instrument line-shape (ILS) and spectral characterization. This paper is organized as follows: Sect. 2 provides a succinct overview of the OCO-2 instrument. Sect. 3 discusses the retrievals based on TVAC data acquired by the OCO- 2 . Section 4 focuses on the fortuitous detection of a $\mathrm{CO}_{2}$-rich plume associated with the Los Angeles urban dome during the TVAC tests and Sect. 5 summarizes the overall work.

The OCO-2 instrument is a three-channel grating spectrometer. It records spectra of the $\mathrm{O}_{2}$ A-band $(0.757-$ $0.775 \mu \mathrm{m}$, full width at half maximum $(\mathrm{FWHM})=0.042 \mathrm{~nm}$, denoted as $\mathrm{O} 2 \mathrm{~A})$, a weak $\mathrm{CO}_{2}$ band $(1.594-1.627 \mu \mathrm{m}$, $\mathrm{FWHM}=0.076 \mathrm{~nm}$, denoted as $\mathrm{WCO} 2$ ) and a strong $\mathrm{CO}_{2}$ band $(2.043-2.087 \mu \mathrm{m}, \mathrm{FWHM}=0.097 \mathrm{~nm}$, denoted as $\mathrm{SCO} 2)$ with eight independent along-slit focal plane array readouts, denoted as footprints (1-8). A common telescope feeds all three spectrometers through a series of beam splitters and re-imagers, with a linear polarizer selecting only the polarization vector perpendicular to the entrance slit. Each spectrometer works in first order of the holographic grating. The system is optically fast (F/1.8) and yields a high signalto-noise ratio (SNR). See Crisp et al. (2004), Pollock et al. 
(2010) and Haring et al. (2008) for a more general description of the OCO instrument and mission.

At each spectrometer's focus, a $1024 \times 1024$ imaging array collects the spectrum, such that one dimension measures field angles along the slit, while the other dimension measures wavelengths. The $\mathrm{O}_{2}$ A-band detector is a silicon (HyViSI) Hawaii-1RG, and the two $\mathrm{CO}_{2}$ detectors are HgCdTe Hawaii-1RG; all were manufactured by Teledyne Scientific and Imaging, LLC. Only 160 of the 1024 pixels in the spatial dimension are used, and sets of $\approx 20$ are averaged onboard to constitute the eight spatial along-slit footprints. In the spectral dimension, four reference pixels are blacked out on each end of the array, leaving 1016 pixels (or channels) per band and footprint. Figure 1 shows an example of the focal plane array $(1016 \times 160$, with block averages of 20 rows in the spatial dimension) readout of all three bands using a direct sun measurement, corresponding to an air mass equivalent to a typical in-space viewing geometry (solar zenith angle of $67^{\circ}$ ). For retrieval purposes, it is important to note that each spectral band (three), footprint (eight) and spectral pixel (1016) has its own characterization; this is in contrast to the FTS instrument onboard GOSAT (Hamazaki et al., 2005; Kuze et al., 2009). The ILS, for instance, is given for each of the $3 \times 8 \times 1016$ detector elements independently, resulting in a 4-dimensional array with the dimensions $3 \times 8 \times 1016 \times 200$ (the ILS is defined for 200 spectral points around the center point).

The OCO-2 instrument was designed to have a spectral sampling of approximately 2.5 detector elements per full width at half maximum (FWHM), in each band, and a spectral resolving power $\lambda / \Delta \lambda$ of approximately 20000 in the $\mathrm{CO}_{2}$ channels and 17000 in the $\mathrm{O}_{2} \mathrm{~A}$ channel. The shape of the OCO-2 ILS function is determined by the slit width, pixel pitch, optical aberrations, diffraction, and detector crosstalk. Details of the ILS as well as spectral and radiometric characterization that are used in this manuscript can be found in Rosenberg et al. (2015), Lee et al. (2015), Day et al. (2011) and O'Dell et al. (2011). Figure 2 shows an example of the OCO-2 ILS for footprint 4 in each band at four different detector array spectral positions. The ILS variation in the spatial direction (footprint) is not as strong as in the spectral direction but still needs to be taken into account for accurate retrievals. As mentioned above, ILS functions are provided in tables that can be interpolated because conventional lineshape functions (e.g., Gaussian, Voigt) cannot fit the shapes well enough for accurate $X_{\mathrm{CO}_{2}}$ retrievals.

\section{Thermal Vacuum Test (TVAC) results}

\subsection{Experimental setup}

During the characterization and calibration of the OCO-2 flight instrument at the JPL, a heliostat was used to direct sunlight onto a diffuser that was viewed by the flight in-

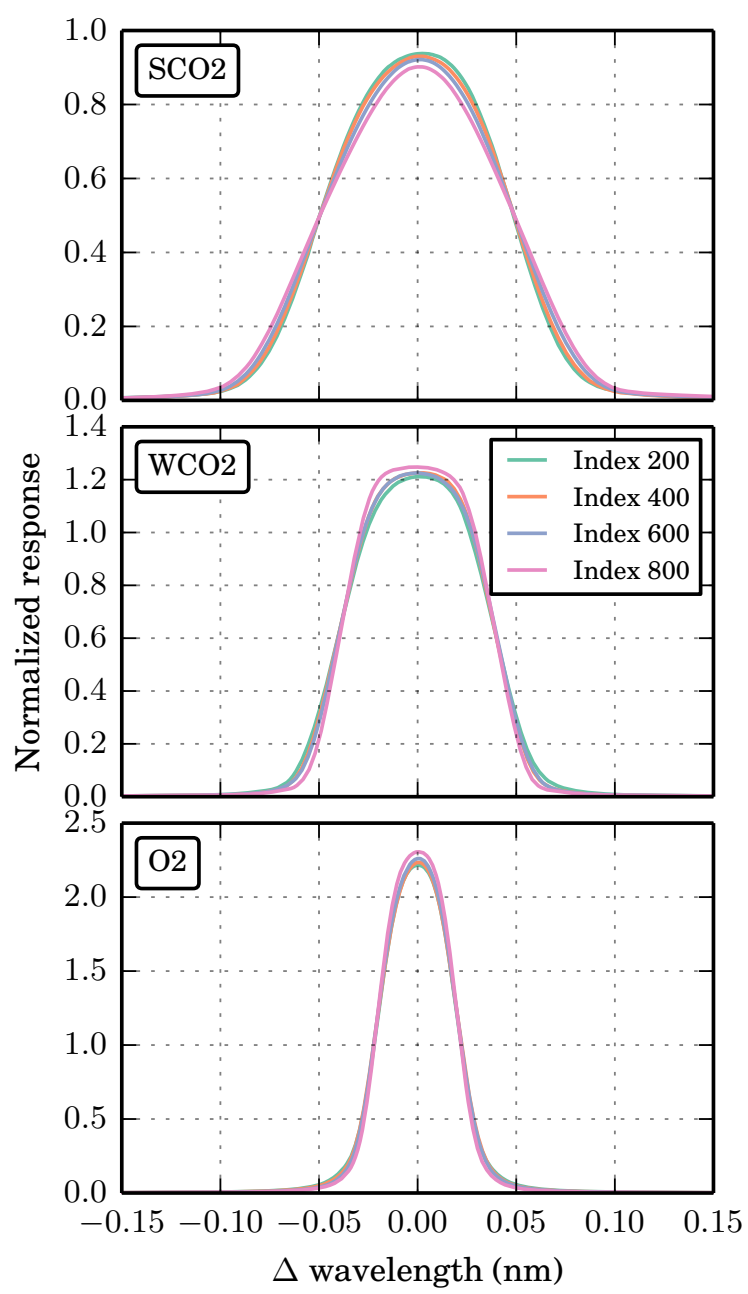

Figure 2. OCO-2 ILSs for a center footprint (\#4) at four different spectral positions for each band independently. FWHM values are about $0.04,0.075$ and $0.1 \mathrm{~nm}$ for the $\mathrm{O} 2, \mathrm{WCO} 2$ and $\mathrm{SCO} 2$ bands, respectively.

strument through a window in the TVAC chamber. Simultaneously, a TCCON instrument located at the same altitude about $200 \mathrm{~m}$ away recorded high-resolution solar spectra through essentially the same atmospheric column. The FTS spectra were acquired at an unapodized resolution of $0.013 \mathrm{~cm}^{-1}$ (45 cm optical path difference), which is approximately 20 times higher than the spectral resolution of the OCO-2 instrument. In addition, the FTS spectra had high SNR and were characterized by a single, well-determined ILS for the entire spectral range (Wunch et al., 2011). Apart from the spectral resolution, there are a few other differences between the two measurements. First, the integration time of the OCO-2 instrument was $0.333 \mathrm{~s}$ (a continuous $3 \mathrm{~Hz}$ measurement) while the FTS required $79 \mathrm{~s}$ to complete a scan. Second, the OCO-2 instrument had a larger field of view (FOV) than the FTS, observing the full solar disk and some of the surrounding sky, while the FTS observed the center 


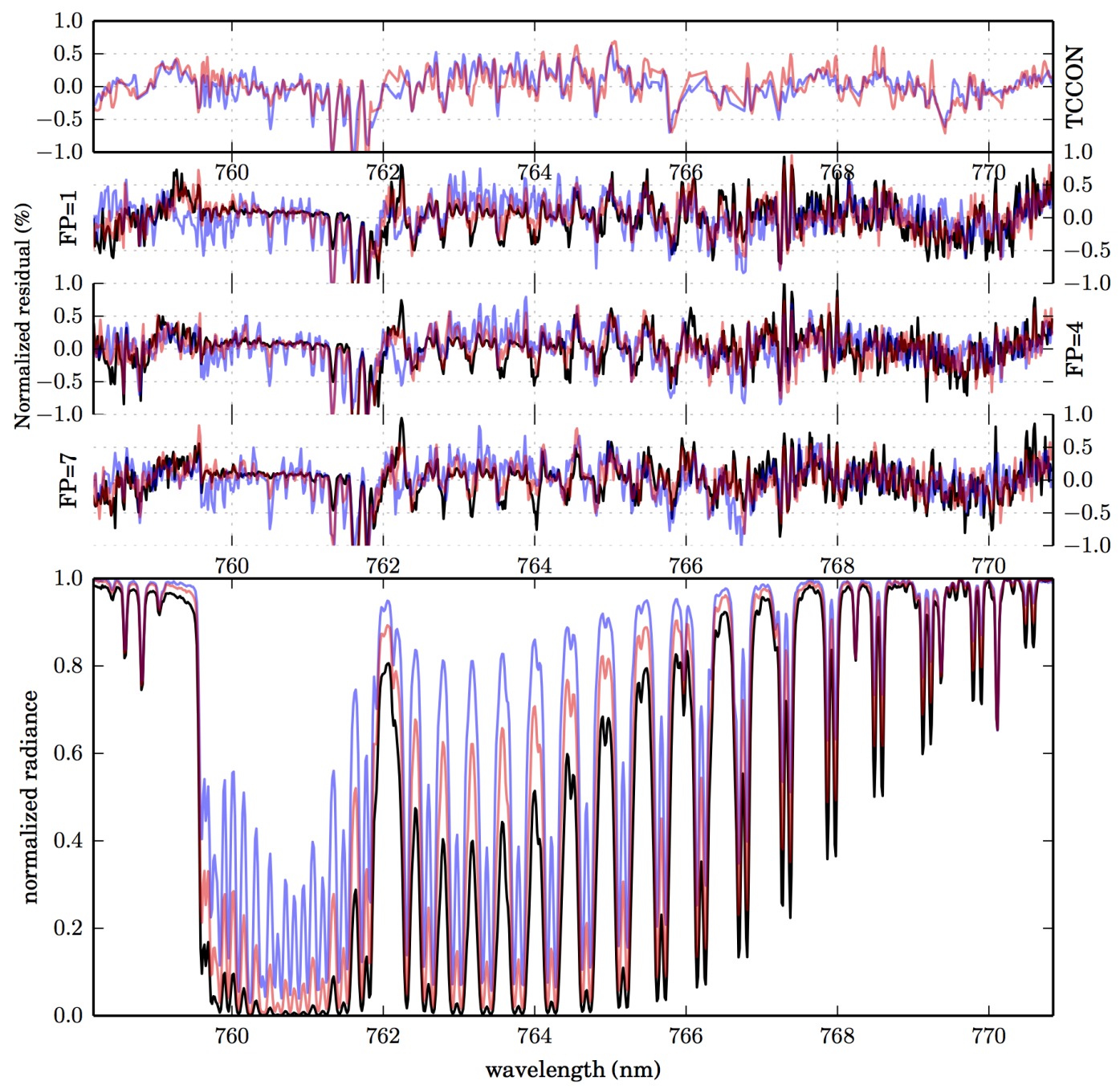

Figure 3. Spectral fits to direct-sun measurements in the O2A band of OCO-2 and TCCON. Normalized radiances from OCO-2 are shown in the bottom panel for three different air masses (low (1.1), typical (2.5) and high (5.1) in blue, red and black, respectively). The middle panels show residuals of fits to normalized spectra, for three OCO-2 footprints, and the top panel shows fit residuals to a high-resolution FTS spectrum of identical air mass, which was convolved and resampled to OCO-2 spectral resolution prior to fitting.

of the solar disk. The solar lines observed by OCO-2 were therefore broadened relative to those observed by the FTS due to Doppler shifts caused by the sun's rotation. For this reason, solar line pixels in the FTS fits were excluded in the following analysis, as we aimed at using exactly the same retrieval setup as for the OCO-2 fits.

\subsection{Spectral fits}

For the spectral fits, we performed total column retrievals of trace gases for each band $(\mathrm{O} 2 \mathrm{~A}, \mathrm{WCO} 2, \mathrm{SCO} 2)$ independently. For this purpose, we used the fast IMAP-DOAS preprocessor in a special up-looking retrieval mode (Frankenberg et al., 2005) using a single temperature and pressure a priori profile for the entire day, which was extracted from the National Centers for Environmental Prediction (NCEP) data, but replacing the surface pressure with the one locally measured at JPL. In this up-looking mode, the IMAP-DOAS algorithm merely fits spectral dispersion, solar shift and continuum baseline as a third-order polynomial, as well as a total column scaling of a pre-defined $\mathrm{O}_{2}, \mathrm{H}_{2} \mathrm{O}$ and $\mathrm{CO}_{2}$ profile shape. Neither surface pressure nor a temperature scaling was retrieved.

In the following, we will show spectral fits for a low (1.1), typical (2.5) and high (5.1) air mass factor (AMF) in blue, red and black, respectively. The "typical" AMF will be very close to the lower end of AMFs encountered during flight, as the AMF in perfect nadir viewing simplifies to $1+1 / \cos (\mathrm{SZA})$ with SZA denoting the solar zenith angle, and the instrument is required to return data at solar zenith angles between $\sim 21.5$ and $85^{\circ}$. To compare the OCO2 spectral fit results (i.e., trace gas columns and fit residuals) 


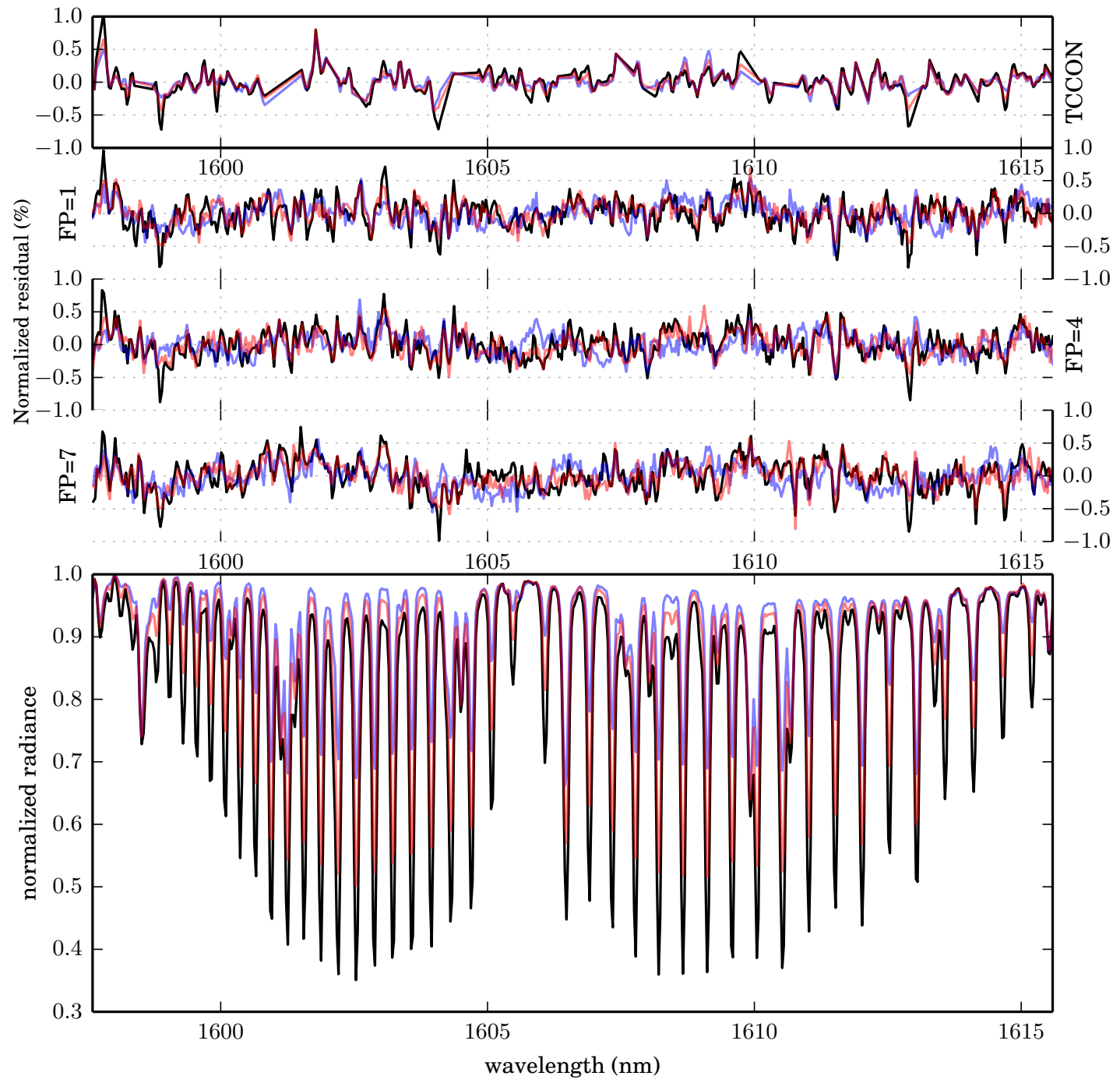

Figure 4. Spectral fits to direct-sun measurements in the WCO2 band of OCO-2 and TCCON (analogous to Fig. 3).

to a high-quality reference, we used collocated FTS spectra and convolved them with the OCO-2 ILS, and performed fits on those spectra using exactly the same fitting routines as for the OCO-2 data. This ensures that differences in the fits are caused neither by differences in spectral resolution nor by specific software setups. Also, convolved spectral residuals of fits to high-resolution (e.g., FTS) data are not equal to residuals of fits to convolved spectra. In addition, solar lines were masked out for the FTS fits since, as noted above, the OCO-2 instrument, in TVAC mode, records full-disk solar spectra while the FTS records narrow FOV disk-centered spectra. In the following, we will display fit residuals in percent, normalized by a representative continuum level radiance, chosen as the maximum signal within each band. For the comparison, we chose OCO-2 and TCCON spectra where the zero-path difference (ZPD) was within a few seconds of the OCO-2 spectra acquisition. For the sake of clarity, we show residuals from only three of the eight footprints. However, we did verify that all footprints behave similarly. For all tests, we used the absorption coefficient (ABSCO) v4.2 spectroscopy database developed for OCO-2 (Thompson et al., 2012).

Spectral fits to the oxygen A-band are shown in Fig. 3. The top panels show normalized residuals of the convolved FTS fits, followed by spectral residuals for 3 OCO-2 footprints (footprints 1, 4 and 7). The bottom panel shows normalized radiances for each air mass. At higher air masses, the R-branch from 760 to $762 \mathrm{~nm}$ saturates almost entirely even at OCO-2 spectral resolution, with near-zero transmission across $1-2 \mathrm{~nm}$ in wavelength range.

In general, fit residuals of the convolved TCCON spectra strongly resemble those of the OCO-2 flight instrument. Given the high SNRs for individual OCO-2 spectra in TVAC mode (600-1200 for all bands), purely noise-driven residu- 


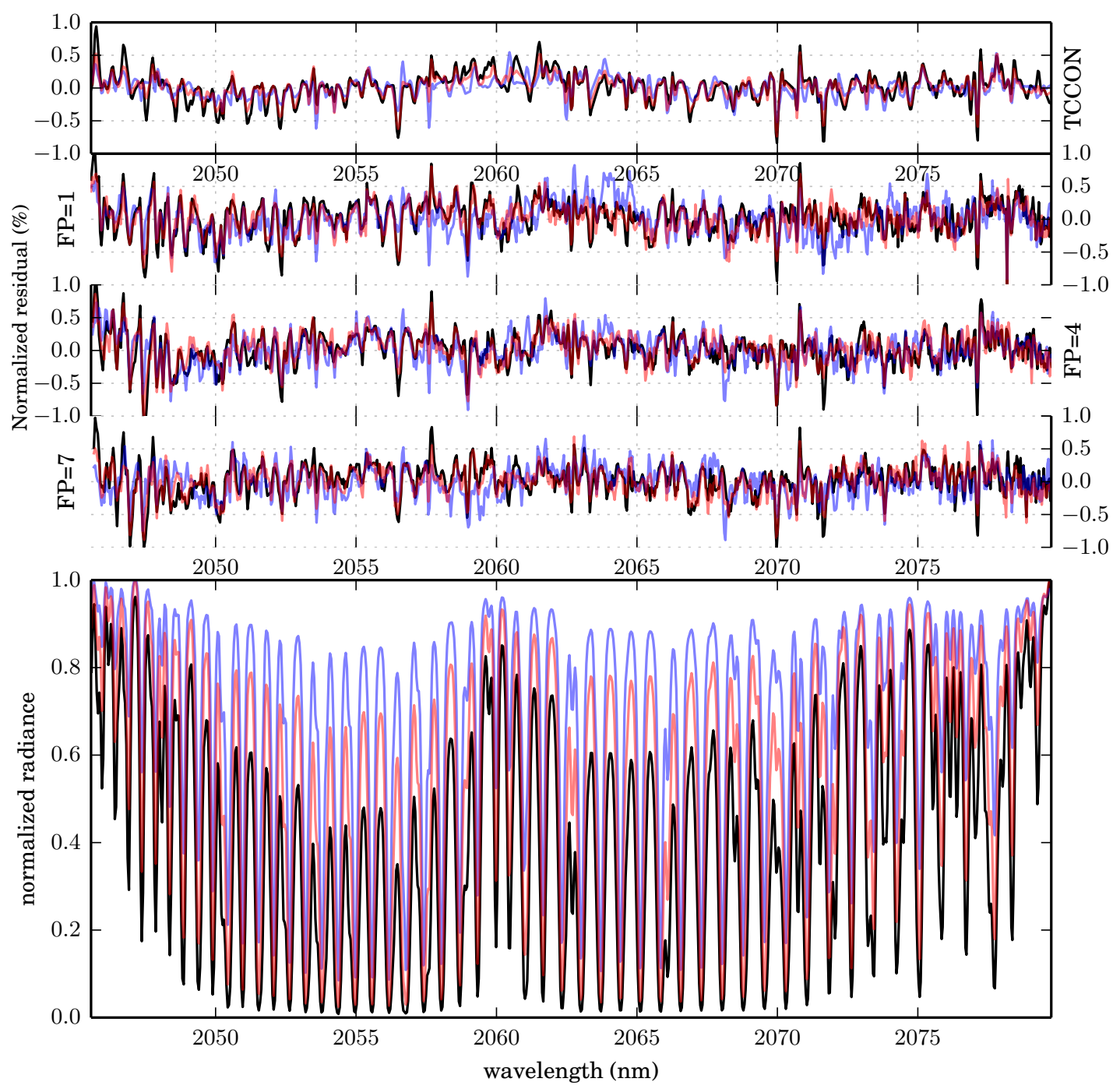

Figure 5. Spectral fits to direct-sun measurements in the SCO2 band of OCO-2 and TCCON (analogous to Fig. 3).

als should be on the order of $0.1-0.2 \%$. Systematic features appear in both TCCON and OCO-2 residuals with maxima of up to $1 \%$. Larger deviations in the R-branch are most likely caused by the choice of the a priori temperature and pressure profile. Given that the two instruments show very similar residuals, we conclude that they are still dominated by errors in the ABSCO tables of the $\mathrm{O}_{2} \mathrm{~A}$-band absorption cross sections, specifically relating to line mixing, line-shape and collision-induced absorption. This can also be seen in the air mass dependence of systematic residuals. At higher air masses, the far line-wing shape becomes more important as line centers are entirely saturated. The periodic structures in the $\mathrm{P}$ branch residuals point to the importance of the line shape as residuals are smaller at lower air mass, at which the far wing line shape becomes less important.

Weak $\mathrm{CO}_{2}$ band (WCO2) fits are shown in Fig. 4. At OCO-2 spectral resolution this band is not saturated even at high air masses. Most of the information content is thus in the line centers as measured by OCO-2, and the continuum can be isolated much more easily from well-separated $\mathrm{CO}_{2}$ lines. Again, we observe residuals that strongly resemble those from TCCON fits (with many solar features masked), pointing to small remaining errors in $\mathrm{CO}_{2}$ absorption cross sections. There is a strong similarity between footprints but, in general, most features are below $0.5 \%$, similar to measurements from the GOSAT satellite as well as TCCON. Some residual features, such as the slope at the short-wavelength edge of the fit window, can also be caused by broad spectral variations in the solar spectrum that are not accurately represented here.

$\mathrm{SCO} 2$ fits are shown in Fig. 5. Line centers at OCO-2 resolution now gradually saturate at higher air mass and the continuum level radiances are harder to define than in the other bands because of overlapping line wings. In general, conclu- 
sions for this band are similar to the other bands, with spectral residuals roughly resembling the TCCON residuals. The only exception may be at the short-wavelength edge where OCO-2 residuals are somewhat higher, potentially caused by either dispersion or ILS changes at the band edge, where the instrument calibration is less well characterized.

In summary, spectral fits to OCO-2 data yield expected fitting results but, as with all other current instruments measuring this spectral range, systematic residuals still appear. Given the high SNR of the OCO-2 instrument, this would result in unrealistically high $\chi^{2}$ values for spectral fits, which is why we will implement a residual fitting technique based on empirical orthogonal functions derived from systematic features as is currently done for data from GOSAT. Using these basis functions, as additional fit parameters, we expect fit residuals to be mostly noise driven, resulting in realistic $\chi^{2}$ values that will help the fit converge, allow us to use $\chi^{2}$ as quality criteria, and result in more realistic a posteriori uncertainty estimates, as the true OCO-2 measurement noise can be provided to the weighted least squares fit.

To determine whether the variability in spectral residuals can be explained by OCO-2 detector noise, we computed the standard deviation of spectral residuals using 2000 OCO2 fits and define this as a surrogate for empirically derived noise in the OCO-2 data. Using the mean of the 2000 radiance spectra as signal level surrogate, we then derive a set of SNR points at varying signal levels within a spectrum, mostly caused by $\mathrm{CO}_{2}$ and $\mathrm{O}_{2}$ absorptions. Figure 6 shows the empirically derived SNR points as well as a typical SNR relationship based on the launch-ready OCO-2 calibration (Rosenberg et al., 2015). There is excellent agreement between calibration curves and empirically derived SNR based on spectral fits. The spread of the individual data points for each band is due to the behavior of the detector, with varying gain coefficients and shot noise in each pixel. This is reflected in the OCO-2 launch-ready calibration curves as well but, for clarity, the theoretical SNR curve of just a typical pixel is shown in transparent lines, and corresponds very well with the empirical noise estimates. After accounting for the systematic residual features, expected $\chi^{2}$ distributions of the residuals are thus attained.

Two advantages of the OCO-2 grating spectrometer are its high dynamic range and relatively low noise at low signal levels. This results in much lower noise levels within deep absorption lines of the $\mathrm{O} 2 \mathrm{~A}$ and the $\mathrm{SCO} 2$ bands than would be possible with space-based FTS data, which exhibit a constant absolute noise level across the entire spectrum (which would yield a straight line through the origin in the SNR vs. signal level curve when plotting individual spectral points within a single acquisition).

\subsection{Footprint dependencies}

The OCO-2 spectrometer records spectra separately in the spatial domain of the focal plane array. To increase SNR,

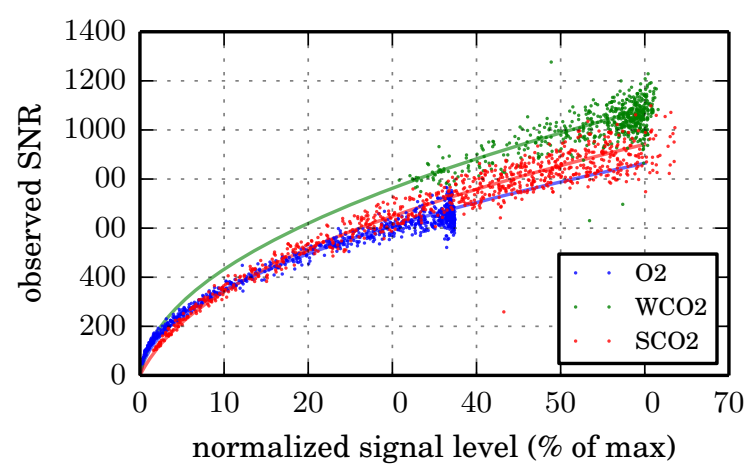

Figure 6. Empirical SNRs of each OCO-2 band derived from the standard deviation of spectral residuals from 2000 separate fits. Solid transparent lines represent the expected OCO-2 SNR for a typical detector pixel, which agrees well with the observed SNR. For each band (O2A, WCO2, SCO2), the maximum observable signal is defined as $7.0 \times 10^{20}, 2.45 \times 10^{20}$ and $1.25 \times$ $10^{20}$ photons s ${ }^{-1} \mathrm{~m}^{-2} \mathrm{sr}^{-1} \mu \mathrm{m}^{-1}$, respectively, where it is taken into account that OCO-2 will only measure one polarization direction (i.e., the maximum signal level in terms of full intensity would be twice as high as provided here).

photons collected in 20 adjacent spatial rows are averaged to define a footprint, hence the 160 spatial detector rows produce eight spatial footprints (see Fig. 1). Given that each footprint spans a considerable fraction of the focal plane array, instrument calibration (most importantly dispersion and ILS) varies from footprint to footprint. With the unprecedented accuracy requirements in the sub $1 \%$ range for atmospheric $\mathrm{CO}_{2}$ measurements, inter-footprint difference in retrieved quantities for imaging spectrometers will cause challenges in using this data.

In Fig. 7, we show differences between the total column $\mathrm{CO}_{2}$ estimates from each footprint and the all-footprint average, for the weak and strong $\mathrm{CO}_{2}$ bands. Two main features are apparent: (1) there is a very small inter-footprint variation but at least six to seven of the footprints agree to within $\pm 0.5 \mathrm{ppm}$; (2) the inter-footprint variations are somewhat unstable and appear to be influenced by how the heliostat is illuminating the OCO-2 instrument, as can be seen by some jumps around 16:00, when the heliostat was realigned. In the test setup, the heliostat was not large enough to fill the entire OCO-2 pupil, which can slightly affect the characteristics (Liebe et al., 2009). This effect, which will not happen in orbit, might change when the heliostat is realigned. Hence, it is not yet clear what fraction of the variation is merely caused by the experimental setup and what is caused by potential small calibration inconsistencies. In orbit, the inter-footprint differences are not expected to vary in time and the variability of $\pm 0.5 \mathrm{ppm}$ can be characterized using in-orbit data and corrected for using constant correction factors. It should be mentioned that the OCO-2 pupil during calibrations using the integrating sphere was fully illuminated. 

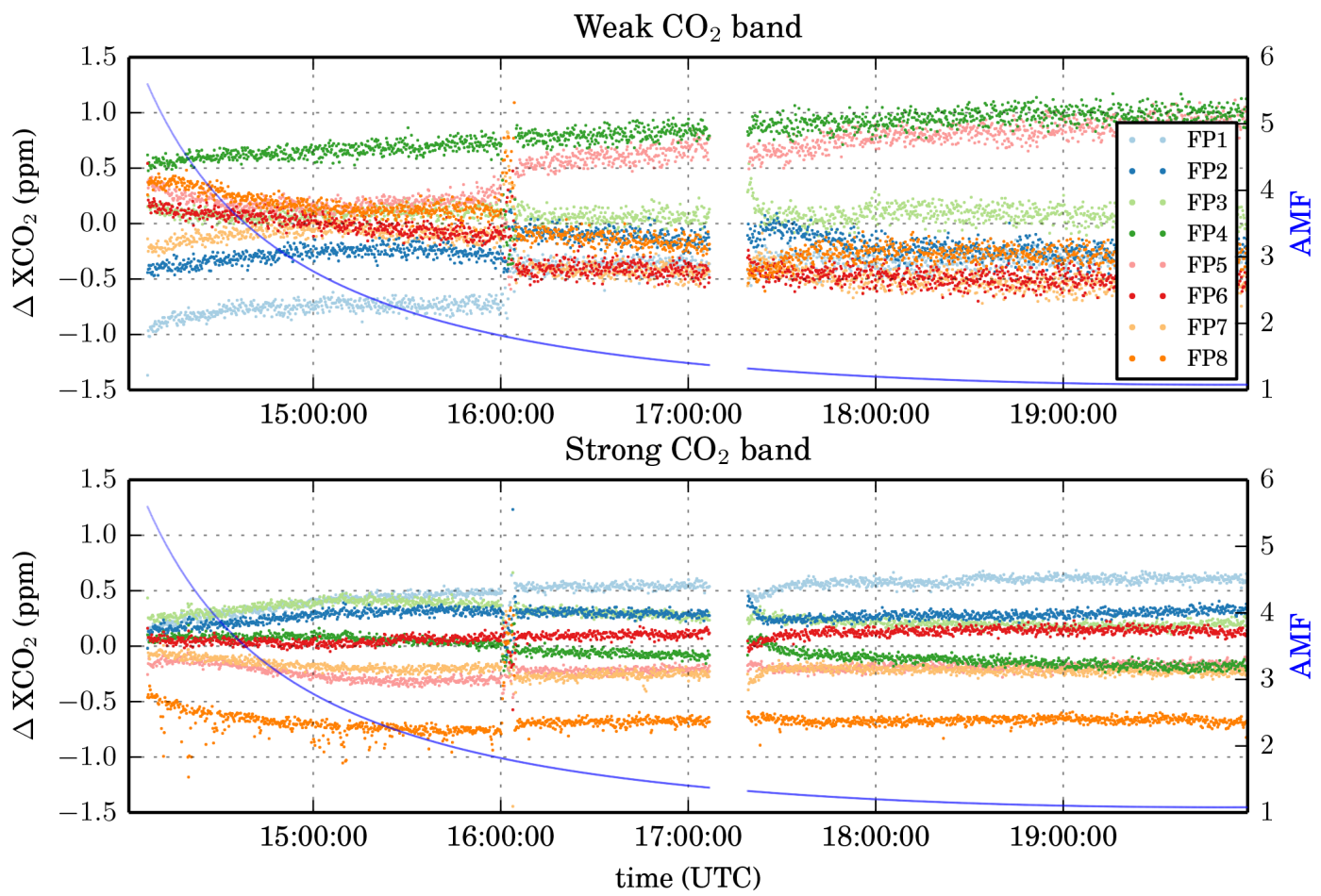

Figure 7. Footprint differences in retrieved $\mathrm{CO}_{2}$ columns using the strong and weak bands from OCO-2 TVAC data acquired on 21 April 2012.

For the pre-launch OCO-2 calibration, two sets of instrument line shapes were tested (Lee et al., 2015), of which one will eventually be implemented for official OCO-2 retrievals. One set is derived from tunable diode laser measurements and a second, enhanced set (see Lee et al., 2015) using direct comparisons against the high-resolution FTS spectra, where the ILS was optimized to best match the FTS data. In Fig. 8, the inter-footprint variations averaged over a longer timeperiod are shown independently for the FTS enhanced ILS (solid lines) and the original laser-based ILS (dashed lines). The impact is not large and mostly affects footprint \#1, at the edge of the FOV. For this particular footprint, both $\mathrm{CO}_{2}$ bands would behave somewhat abnormally but deviate in opposing directions (positive or negative). This could have implications for a joint three-band retrieval. The enhanced ILS largely mitigates this problem to the best possible degree. Once OCO-2 is collecting in-orbit data, both ILS models will be evaluated in order to decide whether the FTS-enhanced ILS mitigated only an issue associated with the TVAC test setup or a real calibration issue. In any case, most footprints agree very well with each other for both choices of ILS and provide confidence, especially given the small fitting residuals discussed earlier. However, even this small footprint dependence will have to be scrutinized since the accuracy requirements of better than $1 \mathrm{ppm}$ are very stringent. This also has implications for future imaging spectrometers with hundreds of spatial pixels, for which a careful per-footprint calibration as for OCO-2 might not be feasible.

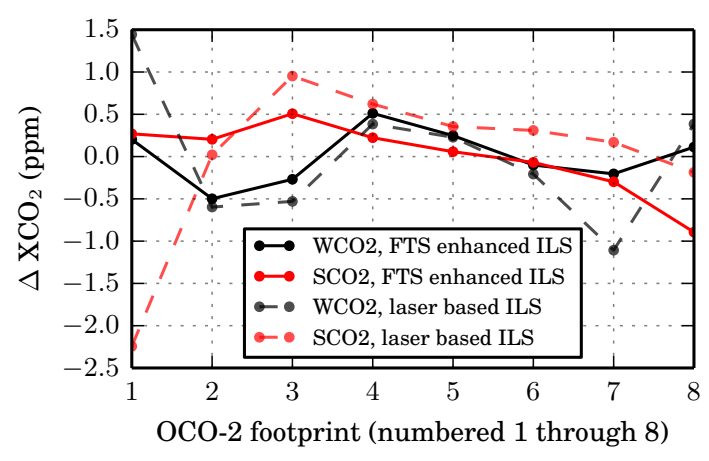

Figure 8. Average of inter-footprint differences in retrieved $\mathrm{CO}_{2}$ columns from OCO-2 using two different sets of instrument line shapes, namely the laser-based ILS and the FTS-enhanced ILS estimate.

\subsection{The Matador test}

The OCO-2 retrieval strategy imposes stringent requirements on knowledge about the detector linearity and dark current. Any imperfections can slightly modify the fractional depths of absorption lines depending on the detector fill level, which can create regional and/or seasonal biases in retrieved $X_{\mathrm{CO}_{2}}$. For OCO, the so-called "Matador test" was devised to detect any apparent non-linearities or dark offsets (O'Dell et al., 2011). 

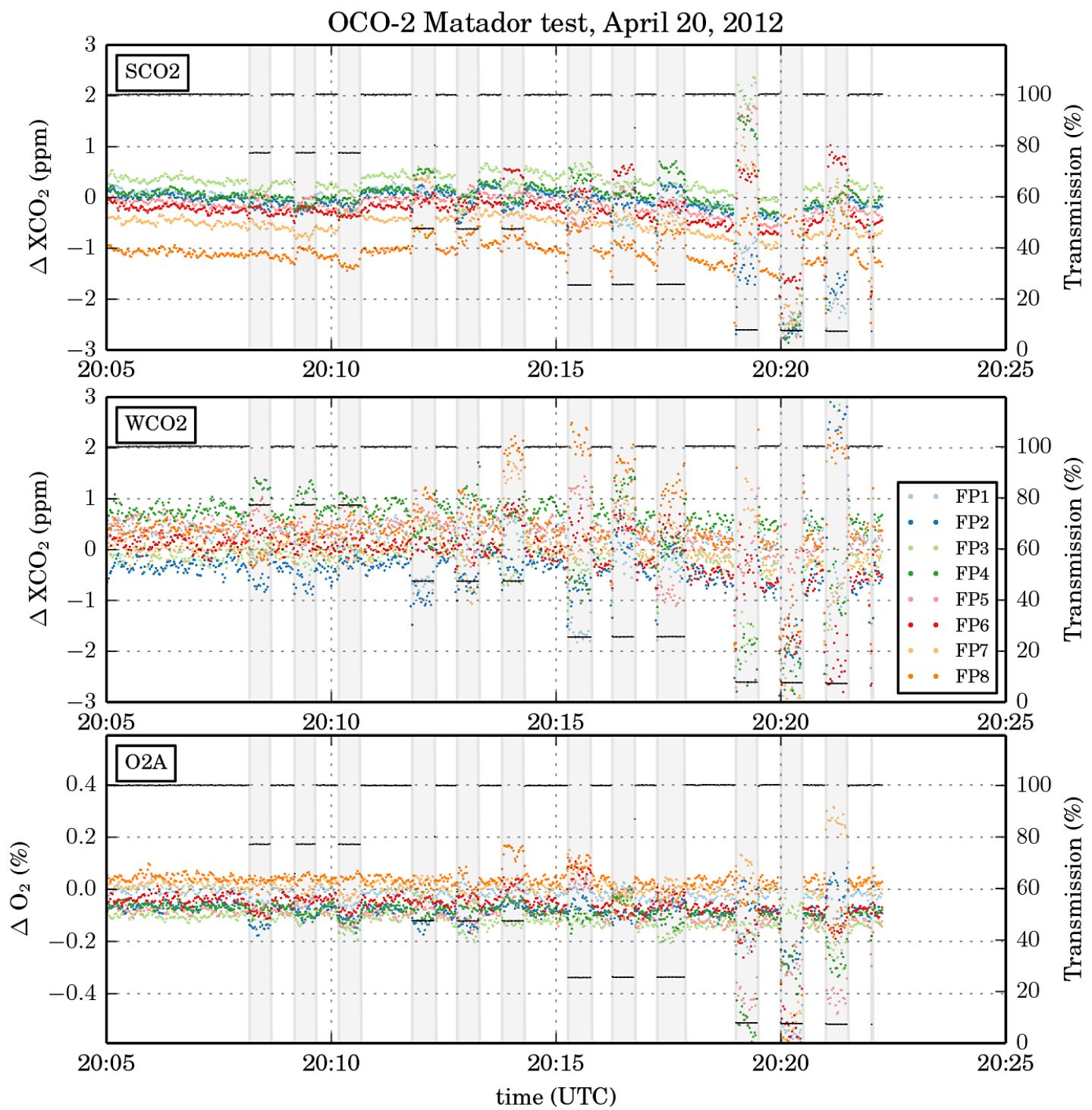

Figure 9. $X_{\mathrm{CO}_{2}}$ (in ppm) and $\mathrm{O}_{2}$ (in \%) differences from a common average in the OCO-2 Matador test, which changed signal levels through inserting aluminum sheets with variable hole density into the light beam. Gray shades indicate the time periods that the aluminum sheets were in the beam and the black horizontal lines indicate the transmission level for each of these tests. Each footprint for both $\mathrm{CO}_{2}$ bands and the $\mathrm{O}_{2}$ band is displayed separately.

The general TVAC setup was used with direct sunlight illuminating a diffuser viewed by the OCO-2 spectrometers via a series of mirrors. For the Matador test, special sheets with varying transmission were optionally inserted into the optical path. To create a spectrally uniform reduction in the solar intensity, aluminum sheets with small holes were fabricated with different hole-densities in a hexagonally packed pattern to create effective transmissions of approximately 75 , 50,25 and $10 \%$. For the test, the sheets were rapidly (and manually) inserted into and removed from the heliostat optical path, hence the name "Matador test" due to the visual analogy to a matador's cape. The test was performed near local noon on 20 April 2012.
Figure 9 shows results for both $\mathrm{CO}_{2}$ bands and the $\mathrm{O}_{2}$ A-band independently, for each footprint, expressed as deviations in $X_{\mathrm{CO}_{2}}$ or $\mathrm{O}_{2}$ column from the average across all footprints at the initial $100 \%$ transmission level (i.e., before the sheets were inserted). The periods with reduced transmission are indicated with a gray background, as each sheet, in descending order of transmission, was inserted and removed three times.

For the 75, 50 and $25 \%$ transmission levels, changes on the order of much less than $0.5 \mathrm{ppm}$ occur. More importantly, deviations are neither consistent across footprints nor consistent across all three independent Matador tests per transmission level. This behavior hints at subtle changes in how the OCO-2 pupil was illuminated for each of these tests, which 


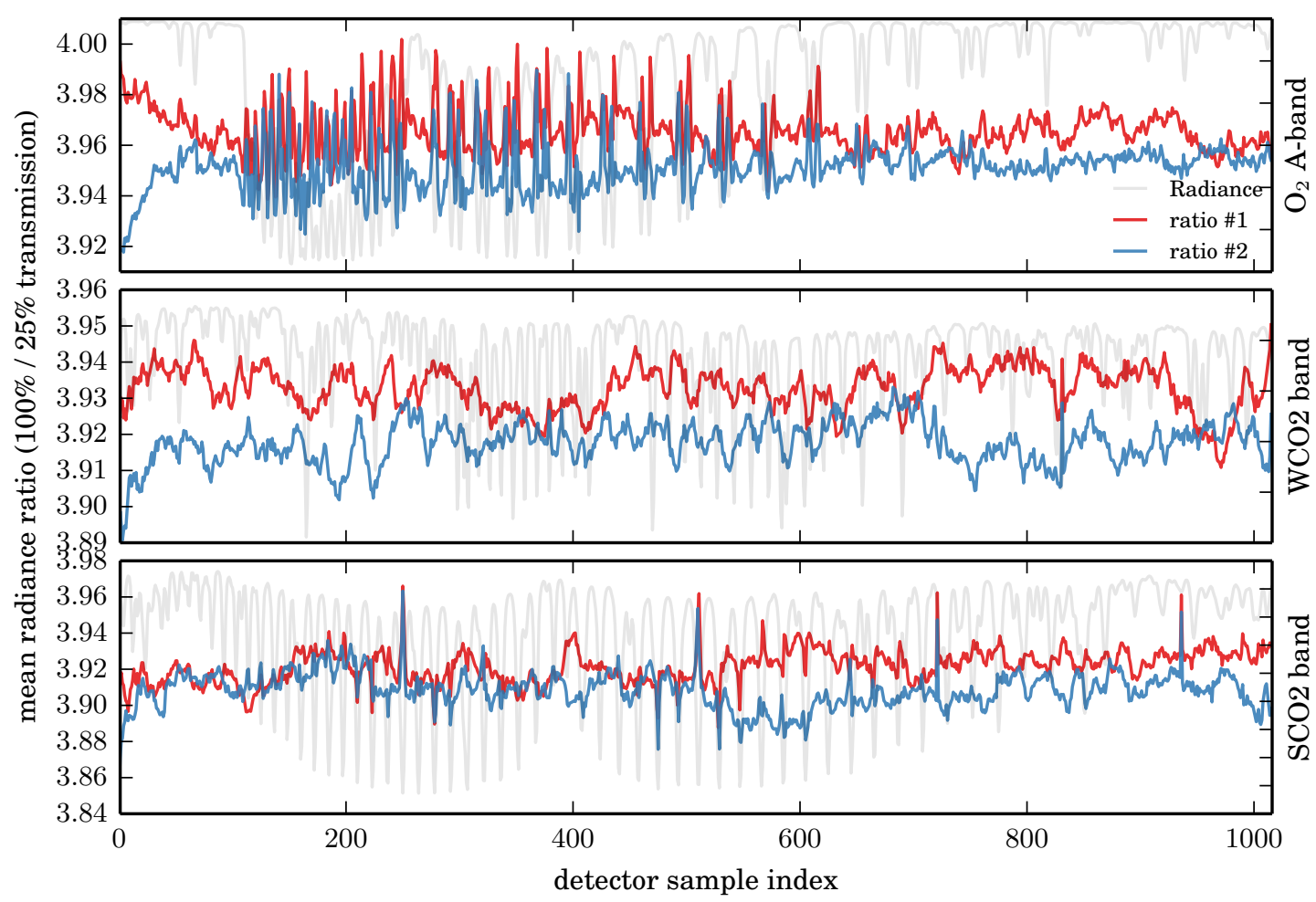

Figure 10. Radiance ratios derived from the Matador tests for footprint \#4. We used the first two $25 \%$ transmission tests recorded between 20:15 and 20:17 UTC and computed three average spectra: one each for for the two periods with the sheet included and one for the full illumination in between the tests. As a visual guide for where the absorption lines are located, a scaled spectrum is plotted in a light gray for each band.

can also cause minor variations in the ILS and, hence, $X_{\mathrm{CO}_{2}}$. This is even more obvious at the $<10 \%$ transmission levels, in which jumps in retrieved $\mathrm{CO}_{2}$ are considerably higher but without any consistent pattern across all three independent tests.

For $\mathrm{O}_{2}$ columns, all footprints agree to about $0.05 \%$, which would translate to better than $0.5 \mathrm{hPa}$ or $0.2 \mathrm{ppm}$ if errors propagate linearly into $X_{\mathrm{CO}_{2}}$. In general, the $\mathrm{O}_{2}$ Aband appeared to be somewhat more consistent than the $\mathrm{CO}_{2}$ bands. It is, however, not clear whether calibration parameters are the cause of the very small variations in the $\mathrm{O}_{2}$ fits or whether these can be merely attributed to the shape of the oxygen absorptions itself, where a lot of information is also coming from the deep Q-R-branch, which is less sensitive to ILS variations as no individual lines are sampled.

As an additional test, we derived radiance ratios of the full illumination level and two separate Matador tests for the $25 \%$ transmission level, as shown in Fig. 10. No absorption features can be detected in the $\mathrm{CO}_{2}$ band ratios, which confirms an accurate OCO-2 calibration in terms of linearity and dark subtraction. However, the two separate tests exhibit ratios with some broad-band features that differ between tests and might be causing slight changes in $X_{\mathrm{CO}_{2}}$ between tests, potentially due to changes in the pupil illumination. Simi-

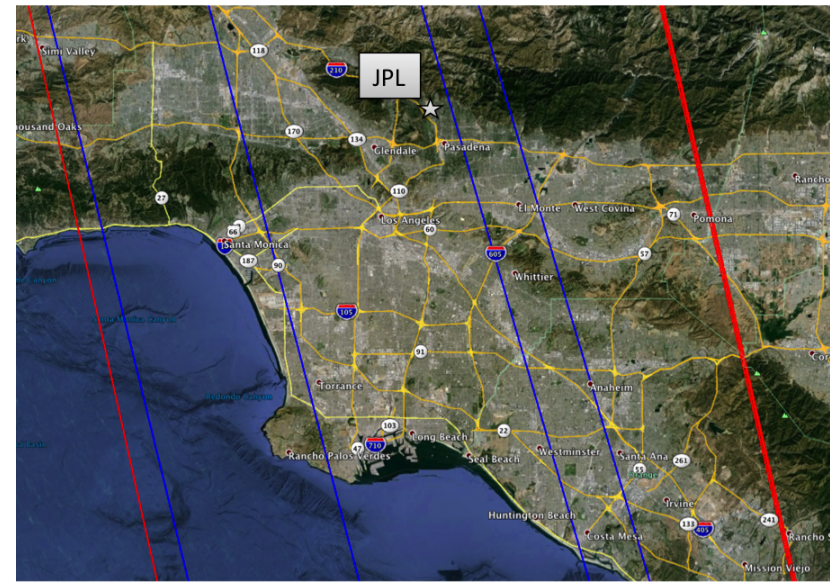

Figure 11. OCO-2 Orbit track simulations for Nadir (red) and Glint (blue), in each season, in the larger Los Angeles area. The nominal target tracks are shown here. These may change slightly, depending on the exact orbit achieved. Nadir repeat cycles of 16 days are close repeats but Glint viewing orbits shift during the year owing to changes in sun-earth geometry. 

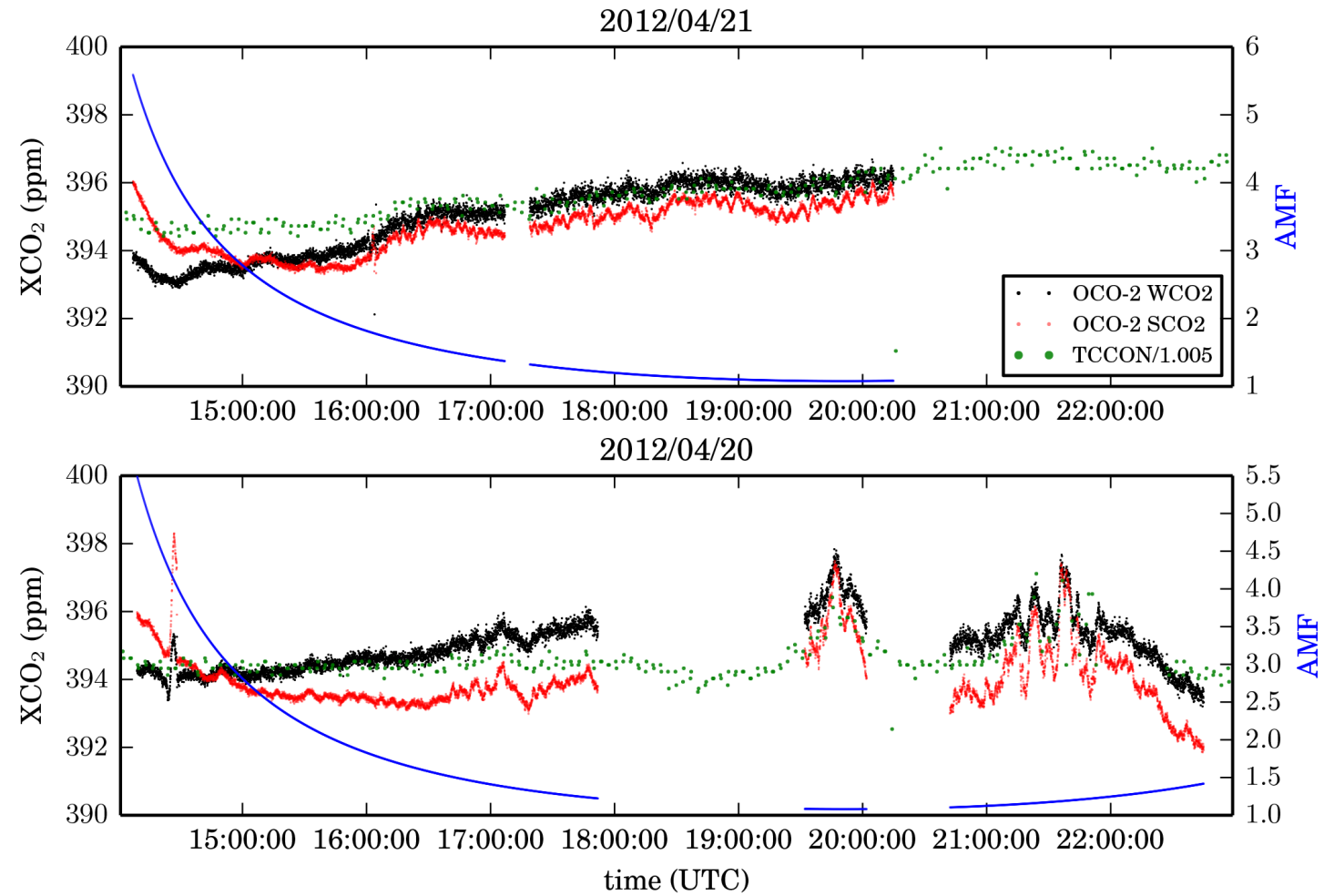

Figure 12. $X_{\mathrm{CO}_{2}}$ time series on 20/21 April 2012 acquired at JPL near Pasadena, CA. Air mass is depicted in blue, SCO2 and WCO2 results in red and black, respectively, and scaled official TCCON data in green. Only every 10th OCO-2 data point is plotted but all eight footprints are averaged.

larly, there appears to be a small change in the ILS for the $\mathrm{O}_{2}$ A-band, most likely also related to the illumination changes.

In summary, all of the three spectral bands show very good consistency, but achieving a near perfect consistency is currently not possible and small calibration factors for each footprint may need to be included for OCO-2. At this stage, we have gained confidence that the OCO- 2 behaves well at variable illumination levels but also that we have obtained the maximum amount of information possible from the TVAC setup. For these pre-launch tests, it is hard to reach the stability in terms of instrument thermal control or illumination that will be achieved in orbit.

\section{Observing the Los Angeles urban dome with the OCO-2 instrument during the TVAC tests}

Apart from the primary mission objective to better understand regional-scale biospheric fluxes, the OCO-2 data can also help us to estimate carbon fluxes from megacities (Kort et al., 2012). Even though its narrow swath will only rarely cross these localized sources, it will have unprecedented spatial resolution and $X_{\mathrm{CO}_{2}}$ sensitivity along the orbit track. Also, the glint repeat cycles will have shifted orbit paths, increasing the chances of obtaining direct cross sections of the urban dome at least once in the OCO-2 mission. An exam-

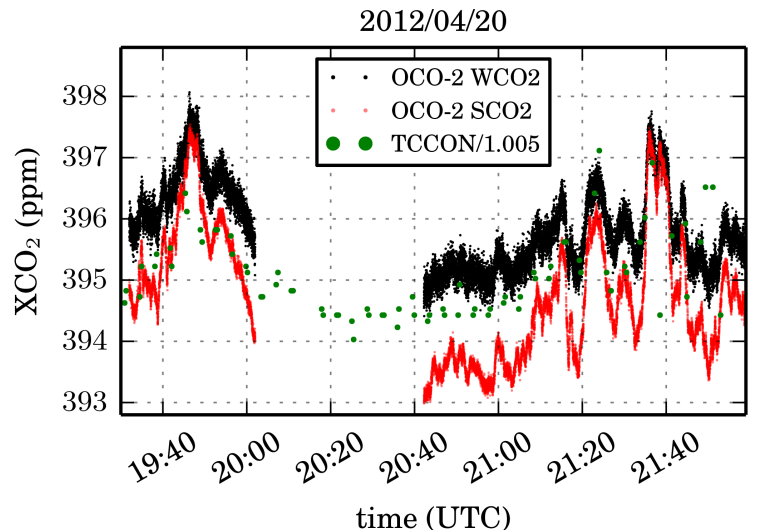

Figure 13. Zoom on the $\mathrm{CO}_{2}$ column time series on 20 April 2012 acquired at JPL. Every OCO-2 data point is plotted and all eight footprints are averaged.

ple of typical OCO-2 orbit paths nearby a large metropolitan area is shown in Fig. 11 for the case of Los Angeles.

In the Los Angeles basin, ground-based studies observed elevated amounts of $\mathrm{CO}_{2}$ and $\mathrm{CH}_{4}$ from total column measurements (Wunch et al., 2009). For $\mathrm{CO}_{2}$, column enhancements measured with a TCCON FTS in 2007/2008 at JPL revealed diurnal changes in $X_{\mathrm{CO}_{2}}$ on the order of up to $4 \mathrm{ppm}$, caused by the Los Angeles urban dome approaching the foothills at JPL around midday. 
Here, we report on the first urban plume observed from the OCO-2 even before launch. Figure 12 shows time series for 20/21 April of $\mathrm{CO}_{2}$ column retrievals using the $\mathrm{WCO} 2$ and $\mathrm{SCO} 2$ bands of OCO-2 as well as the TCCON FTS retrievals located nearby (Wunch et al., 2011). For TCCON retrievals, we extracted the official $X_{\mathrm{CO}_{2}}$ values retrieved using the latest GGG2012 data version (https://tccon-wiki.caltech.edu/Network_Policy/Data_ Use_Policy/Data_Description). These data are based on two weak $\mathrm{CO}_{2}$ bands, one of which is identical with the OCO-2 $\mathrm{WCO} 2$ band. $\mathrm{CO}_{2}$ columns are scaled by retrievals of dry air, obtained from fits to the oxygen band at $1.27 \mu \mathrm{m}$. The official TCCON data applies both an AMF correction and a scaling factor to bring the measured $X_{\mathrm{CO}_{2}}$ onto the NOAA standard $\mathrm{CO}_{2}$ scale as used for the in situ networks.

For OCO-2, all eight footprints have been averaged but no further smoothing has been applied. Owing to the strengths of absorption features in the $\mathrm{SCO} 2$ band, retrieval scatter in the strong band is substantially lower than for the weak band. TCCON data was divided by 1.005 , indicating a small but consistent offset between the official TCCON results and OCO-2. The $X_{\mathrm{CO}_{2}}$ observed on these two TVAC days exhibits substantially different behavior. On 21 April, a relatively smooth diurnal cycle, with maxima around solar noon, was observed with an overall amplitude of about $2 \mathrm{ppm}$ in $X_{\mathrm{CO}_{2}}$. Some short-term features, such as the increase at 16:00 or the swings around 18:30 and 19:30, can be clearly observed in both TCCON and OCO-2, while the latter allows for a much faster sampling in time due to the $3 \mathrm{~Hz}$ readout rate. This also allows us to sample the very erratic behavior seen on 20 April. This day, interrupted by Matador tests around local noon, shows very high-frequency variations of observed $\mathrm{CO}_{2}$ starting around 20:00 UTC. Figure 13 shows a zoom plot into this specific anomalous time-period with high-frequency variability unresolved by the TCCON sampling, but similar general features observed by both instruments. This behavior suggests the edge of the urban dome moving in and out of the observation site. Swings can be up 3-4 ppm within a 5-10 min period, as is observed at about 21:40 UTC. Assuming typical wind-speeds of $5-10 \mathrm{~m} \mathrm{~s}^{-1}$, these temporal scales correspond to spatial scales of 1.5$3 \mathrm{~km}$, which will be matched by the in-orbit OCO-2. In other words, spatial gradients of about 2-4 ppm within a few kilometers can be expected in strongly emitting areas such as Los Angeles. In addition, cross sections of the urban dome can be mapped with the OCO-2 with the caveat of a more complex retrieval from space, where retrievals are based on backscattered radiation and have to take aerosols into account. However, $1 \%$ changes in $X_{\mathrm{CO}_{2}}$ as observed here are substantial and should be differentiable from light-path modifications within the urban dome.

Another feature is apparent in the time series: the amplitude of the variation in the weak band is smaller than in the strong band. This could be caused by differences in the averaging kernels (AK) of both channels in total column scaling mode. The AK of the strong band, with more saturation, typically peaks at much higher values near the surface if a simple total column fit is applied. Thus, $\mathrm{CO}_{2}$ variability at the surface will be more amplified in the $\mathrm{SCO} 2$ band retrievals, which is well reflected in the OCO-2 measurements and is another hint at boundary-layer $\mathrm{CO}_{2}$ enhancements.

\section{Conclusions}

In this work, we performed spectral fits on calibrated OCO2 data obtained during thermal vacuum tests at the JPL in April 2012. Direct sunlight was fed to the OCO-2 instrument via a heliostat and data could be compared against a high-quality reference taken from an FTS located in close proximity. OCO-2 spectra were calibrated with the launchready calibration version from the OCO-2 instrument team. We performed the first $\mathrm{CO}_{2}$ and $\mathrm{O}_{2}$ total column retrievals from the OCO-2, providing a high-level evaluation and reference for the expected OCO-2 instrument performance. We find that spectral residuals are still mostly dominated by systematic features but that these are also apparent in other measurements, including high-resolution FTS data. Hence, most of the systematic features can be attributed to uncertainties in the spectroscopy. We also find that OCO-2 footprints agree very well with each other: better than $1 \mathrm{ppm}$. There is a small time-dependence on the footprint dependency, which is probably related to the specific test setup and would thus not occur in space.

A strong $\mathrm{CO}_{2}$ plume from the Los Angeles metropolitan area (3-4 ppm enhancement in the total column average) was observed on 20 April 2012, when the plume caused very high-frequency $(5-10 \mathrm{~min})$ swings of $\mathrm{CO}_{2}$ at the JPL site. These are indicative of strong spatial gradients in $X_{\mathrm{CO}_{2}}$, which will be very helpful in characterizing localized carbon emissions when OCO-2 orbit tracks passes over such regions.

In summary, the OCO-2 instrument performed very well on the ground and none of the spectral fits calculated in this work showed anything unexpected. The team is now eagerly waiting for in-space OCO-2 data.

Acknowledgements. The research described in this paper was carried out by the Jet Propulsion Laboratory, California Institute of Technology, under a contract with the National Aeronautics and Space Administration. We would like to acknowledge all the JPL employees who worked tirelessly to acquire the OCO-2 TVAC data. The TCCON instrument was built by the California Institute of Technology with support from NASA's OCO-2 project. TCCON data used in this analysis are available at https://tccon-wiki.caltech.edu/Network_Policy/Data_Use_Policy. The TCCON instrument used here is currently operating at the NASA Armstrong Flight Research Center in Edward, California. Government sponsorship acknowledged.

Edited by: D. Griffith 


\section{References}

Bösch, H., Toon, G., Sen, B., Washenfelder, R., Wennberg, P., Buchwitz, M., de Beek, R., Burrows, J., Crisp, D., and Christi, M.: Space-based near-infrared $\mathrm{CO}_{2}$ measurements: Testing the Orbiting Carbon Observatory retrieval algorithm and validation concept using SCIAMACHY observations over Park Falls, Wisconsin, J. Geophys. Res., 111, 0148-0227, 2006.

Butz, A., Hasekamp, O. P., Frankenberg, C., and Aben, I.: Retrievals of atmospheric $\mathrm{CO}_{2}$ from simulated space-borne measurements of backscattered near-infrared sunlight: accounting for aerosol effects, Appl. Optics, 48, 3322-3336, 2009.

Crisp, D., Atlas, R. M., Breon, F.-M., Brown, L. R., Burrows, J. P., Ciais, P., Connor, B. J., Doney, S. C., Fung, I. Y., Jacob, D. J., Miller, C. E., O'Brien, D., Pawson, S., Randerson, J. T., Rayner, P., Salawitch, R. J., Sander, S. P., Sen, B., Stephens, G. L., Tans, P. P., Toon, G. C., Wennberg, P. O., Wofsy, S. C., Yung, Y. L., Kuang, Z., Chudasama, B., Sprague, G., Weiss, B., Pollock, R., Kenyon, D., and Schroll, S.: The orbiting carbon observatory (OCO) mission, Adv. Space Res., 34, 700-709, 2004.

Day, J. O., O’Dell, C. W., Pollock, R., Bruegge, C. J., Rider, D., Crisp, D., and Miller, C. E.: Preflight spectral calibration of the Orbiting Carbon Observatory, IEEE T. Geosci. Remote Sens., 49, 2793-2801, 2011.

Frankenberg, C., Platt, U., and Wagner, T.: Iterative maximum a posteriori (IMAP)-DOAS for retrieval of strongly absorbing trace gases: Model studies for $\mathrm{CH} 4$ and $\mathrm{CO} 2$ retrieval from near infrared spectra of SCIAMACHY onboard ENVISAT, Atmos. Chem. Phys., 5, 9-22, doi:10.5194/acp-5-9-2005, 2005.

Hamazaki, T., Kaneko, Y., Kuze, A., and Kondo, K.: Fourier transform spectrometer for greenhouse gases observing satellite (GOSAT), in: Proceedings of SPIE, Vol. 5659, p. 73, 2005.

Haring, R. E., Pollock, R., Sutin, B. M., Blakley, R., Scherr, L. M., and Crisp, D.: Fabrication and assembly integration of the orbiting carbon observatory instrument, Proc. SPIE, 7082, 708213, doi:10.1117/12.796289, 2008.

Kort, E. A., Frankenberg, C., Miller, C. E., and Oda, T.: Space-based observations of megacity carbon dioxide, Geophys. Res. Lett., 39, L17806, doi:10.1029/2012GL052738, 2012.

Kuze, A., Suto, H., Nakajima, M., and Hamazaki, T.: Thermal and near infrared sensor for carbon observation Fourier-transform spectrometer on the Greenhouse Gases Observing Satellite for greenhouse gases monitoring, Appl. Optics, 48, 6716-6733, 2009.

Lee, R. A. M., et al.: Preflight Spectral Calibration of the Orbiting Carbon Observatory-2, in preparation, 2015.
Liebe, C. C., Pollock, R., Hannah, B., Bartman, R., Radulescu, C., Rud, M., and Esposito, J. A.: System for establishing best focus for the Orbiting Carbon Observatory instrument, Opt. Eng., 48, 073605, doi:10.1117/1.3180867, 2009.

O’Dell, C. W., Day, J. O., Pollock, R., Bruegge, C. J., O’Brien, D. M., Castano, R., Tkatcheva, I., Miller, C. E., and Crisp, D.: Preflight radiometric calibration of the orbiting carbon observatory, IEEE T. Geosci. Remote Sens., 49, 2438-2447, 2011.

O’Dell, C. W., Connor, B., Bösch, H., O’Brien, D., Frankenberg, C., Castano, R., Christi, M., Eldering, D., Fisher, B., Gunson, M., McDuffie, J., Miller, C. E., Natraj, V., Oyafuso, F., Polonsky, I., Smyth, M., Taylor, T., Toon, G. C., Wennberg, P. O., and Wunch, D.: The $\mathrm{ACOS} \mathrm{CO}_{2}$ retrieval algorithm - Part 1: Description and validation against synthetic observations, Atmos. Meas. Tech., 5, 99-121, doi:10.5194/amt-5-99-2012, 2012.

Pollock, R., Haring, R. E., Holden, J. R., Johnson, D. L., Kapitanoff, A., Mohlman, D., Phillips, C., Randall, D., Rechsteiner, D., Rivera, J., Rodriguez, J. I., Schwochert, M. A., and Sutin, B. M.: The Orbiting Carbon Observatory instrument: performance of the OCO instrument and plans for the OCO-2 instrument, Proc. SPIE, 7826, 78260W, doi:10.1117/12.865243, 2010.

Rosenberg, R., Maxwell, S., Johnson, B., and Pollock, R.: Preflight Radiometric Calibration of the Orbiting Carbon Observatory-2, in preparation, 2015.

Thompson, D. R., Benner, D. C., Brown, L. R., Crisp, D., Devi, V. M., Jiang, Y., Natraj, V., Oyafuso, F., Sung, K., Wunch, D., Castaño, R., and Miller, C. E.: Atmospheric validation of high accuracy $\mathrm{CO}_{2}$ absorption coefficients for the OCO-2 mission, J. Quant. Spectrosc. Ra., 113, 2265 - 2276, doi:10.1016/j.jqsrt.2012.05.021, 2012.

Washenfelder, R. A., Toon, G. C., Blavier, J.-F., Yang, Z., Allen, N. T., Wennberg, P. O., Vay, S. A., Matross, D. M., and Daube, B. C.: Carbon dioxide column abundances at the Wisconsin Tall Tower site, J. Geophys. Res.-Atmos., 111, D22305, doi:10.1029/2006JD007154, 2006.

Wunch, D., Wennberg, P. O., Toon, G. C., Keppel-Aleks, G., and Yavin, Y. G.: Emissions of greenhouse gases from a North American megacity, Geophys. Res. Lett., 36, L15810, doi:10.1029/2009GL039825, 2009.

Wunch, D., Toon, G. C., Blavier, J.-F. L., Washenfelder, R. A., Notholt, J., Connor, B. J., Griffith, D. W. T., Sherlock, V., and Wennberg, P. O.: The Total Carbon Column Observing Network, Philos. T. Roy. Soc. A, 369, 2087-2112, doi:10.1098/rsta.2010.0240, 2011. 